Pirineos.Revista de Ecología de Montaña Vol. 166, 135-153

Jaca, Enero-Diciembre, 2011

ISSN: 0373-2568

eISSN: 1988-4281

doi: 10.3989 / Pirineos.2011.166007

\title{
VEINTE AÑOS DE SEGUIMIENTO POBLACIONAL DE UNGULADOS SILVESTRES DE ARAGÓN
}

\author{
J. Marco ${ }^{1}$, J. Herrero², M. A. Escudero ${ }^{1}$, O. Fernández-Arberas ${ }^{3}$, \\ J. FERRERES ${ }^{1}$, A. GARCÍA-SERRANO ${ }^{3}$, A. GIMÉNEZ-ANAYA ${ }^{3}$, J. L. LABARTA ${ }^{1}$, \\ L. MonRABAL ${ }^{1} \&$ C. Prada ${ }^{3}$ \\ 1 Ebronatura S.L. C/del Arpa, 6 (local). E-50003 Zaragoza \\ javiermarco@ebronatura.com \\ 2 Área de Ecología. Departamento de Agricultura y Economía Agraria. \\ Escuela Politécnica Superior de Huesca. Universidad de Zaragoza. E-22071 Huesca \\ herreroj@unizar.es \\ 3 Ega Consultores. Sierra de Vicort, 31, 1. A. E-50003 Zaragoza.egasl@arrakis.es
}

\begin{abstract}
The remarkable increase of wild ungulates populations in Aragon during the last 50 years, both in numbers and distribution, has made population monitoring a need for effective management of these species. In parallel to their raising numbers, problems related to crop damage and collisions with vehicles are growing. In this situation there has been a shift in the management goals from conservation to population control. Different methods have been used in the surveys: postal enquiries for distribution, battues, distance sampling, night spotlight surveys and indirect ones based on transect and plot counts of pellet groups. Surveys started in 1985 for red deer, 1990 for wild boar and Iberian wild goat, 1995 for chamois and 2002 for roe deer, gradually becoming a population monitoring program. The surveys are carried out by rangers and wildlife consultants under the coordination and supervision of the Fish and Game Service of the Aragon Government. The ungulate populations are still growing (wild boar, wild goat, red and roe deer), are relatively stabilised (fallow deer, feral goat), fluctuate due to diseases (chamois) or show small populations limited to fenced states (Barbary sheep, mouflon). Hunting bags went from 1,100 to 36,000 during an eleven-year period (1990-2009): 303 chamois, 1,173 wild goats, 2,141 roe deer, 2,424 red deer and 29,595 wild boars, showing a trend which is still increasing. The main tool is therefore hunting quota and its verification through hunting bags declarations, damages to crops and car crashes, and population trend and abundance.
\end{abstract}

Keywords: Capra pyrenaica; Capra hircus; Capreolus capreolus; Cervus elaphus; Dama dama; Ovis aries; Sus scrofa; Ammotragus laervia; hunting bags. 
RESUMEN.- La importante expansión de las poblaciones de ungulados silvestres en Aragón desde los años 50 del siglo XX hasta la actualidad, tanto en superficie ocupada como en abundancia de ejemplares, ha hecho necesario el seguimiento de las mismas para su adecuada gestión. Su incremento en las dos últimas décadas, junto con el importante aumento de daños agrícolas y colisiones con vehículos, ha motivado un importante cambio en los criterios gestores; pasando de la conservación y fomento al control e incluso reducción de efectivos. Los métodos utilizados para el seguimiento de las poblaciones fueron: encuestas quincenales de distribución, batidas, muestreos de distancia, captura - recaptura, fareos nocturnos y muestreos indirectos basados en el conteo de grupos de excrementos en transectos y parcelas fijas. Los seguimientos se comenzaron a realizar en 1985 en el caso del ciervo, 1990 en el jabali y cabra montesa, 1995 en el sarrio y 2002 en el corzo, habiéndose convertido paulatinamente en una verdadera monitorización poblacional. Los seguimientos son realizados por Agentes de Protección de la Naturaleza del Gobierno de Aragón y asistencias técnicas externas, bajo la supervisión y coordinación de los responsables administrativos. Los resultados obtenidos muestran que, algunas poblaciones continúan su expansión e incremento (jabalí, ciervo, corzo, cabra montesa), otras se encuentran relativamente estabilizadas (gamo, cabra doméstica asilvestrada), y unas pocas fluctúan debido a enfermedades (sarrio) o muestran presencias testimoniales prácticamente reducidas a cercados (arruí, muflón). Las capturas declaradas han pasado de 1.100 a 36.000 en 11 años (1999-2009): 303 sarrios, 1.173 cabras montesas, 2.141 corzos, 2.424 ciervos y 29.595 jabalies, con una tendencia al incremento que aún no ha finalizado. El instrumento fundamental por lo tanto son los cupos de caza y su verificación se realiza a partir de las declaraciones de capturas, los daños a la agricultura y accidentes automovilísticos y la tendencia y abundancia poblacional.

Palabras clave: Capra pyrenaica; Capra hircus; Capreolus capreolus; Cervus elaphus; Dama dama; Ovis aries; Sus scrofa; Ammotragus laervia; capturas cinegéticas.

\section{Introducción}

Los ungulados silvestres han sufrido una importante expansión e incremento demográfico en toda Europa durante las últimas décadas, pasando en gran medida de áreas marginales a ocupar gran parte del territorio (Apollonio et al., 2010). Hoy juegan un papel importante como consumidores primarios, especies cinegéticas y origen de daños agrícolas y a los vehículos. En Aragón sus poblaciones son objeto de seguimiento demográfico y sanitario y la información obtenida marca la gestión desarrollada, particularmente sobre los cupos de extracción. Las especies y poblaciones presentes son: introducidas (gamo Dama dama, arrui Ammotragus laervia); asilvestradas (muflón Ovis aries y cabra doméstica asilvestrada Capra hircus) y autóctonas (jabalí Sus scrofa, sarrio Rupicapra p.pyrenaica, cabra montesa Capra pyrenaica, ciervo Cerous elaphus y corzo Capreolus capreolus). 
Los daños a los cultivos agrícolas son pagados por la administración autónoma en Reservas de Caza y Áreas Naturales Protegidas y ascienden a $180.000 €$ en 2008 y 90.000 en 2009, constituyendo el principal problema de gestión, sobre todo para el caso de corzos y ciervos y en menor medida, de cabra montesa. Los atropellos también van en aumento, suponiendo para el erario público un coste aproximado de $500.000 €$ en 2009 y una cantidad también importante, pero no conocida en la actualidad, pagada por los cotos.

Los resultados de estos seguimientos permiten establecer anualmente cupos de capturas adaptados a las posibilidades poblacionales, sobre la base de datos actualizados, sin por ello perder de vista otros condicionantes de naturaleza antrópica que puedan influir, en zonas y momentos concretos, los criterios de asignación de dichos cupos.

En este trabajo pretendemos mostrar por primera vez en su conjunto los resultados básicos de más de 20 años de seguimientos demográficos de ungulados silvestres en Aragón, sintetizando la información disponible sobre distribución, abundancia y capturas de las diferentes especies y poblaciones, base de las medidas de gestión adoptadas.

\section{2. Área de estudio}

El área de estudio la conforma la Comunidad Autónoma de Aragón con $47.669 \mathrm{~km}^{2}$ y 1.269 .027 habitantes en 2006. El 70\% de la población vive en la provincia de Zaragoza y aproximadamente la mitad del total en su capital, Zaragoza, existiendo grandes superficies con densidades inferiores a los 10 habitantes $\mathrm{km}^{-2}$. Los municipios rurales presentan un perfil demográfico determinado por la emigración y el envejecimiento. El territorio puede dividirse en tres grandes unidades de relieve: el Valle Medio del Ebro; el Pirineo y Prepirineo y el Sistema Ibérico (Sampietro, 2000; De la Riva, 1997).

En el Pirineo las precipitaciones anuales se sitúan por debajo de los 800 $\mathrm{mm}$ sólo en las zonas bajas de los grandes ríos. La temperatura media anual es inferior a $12^{\circ}$. El relieve impone un gran número de variaciones locales, entre las que destacan la alternancia entre solana y umbría, las inversiones térmicas y el encajonamiento de los vientos. La cubierta vegetal, mayoritariamente incluida en el dominio eurosiberiano, denota la transición hacia el mediterráneo. La sucesión altitudinal es clara: escasos pastizales alpinos, piso subalpino muy transformado en matorrales y pastos alpinizados, piso montano (hayedos, abetos, fresnos y extensos pinares de Pinus sylvestris y piso submediterráneo (quejigo Quercus faginea y encina Quercus ilex, con gran deforestación para uso agrícola y ganadero). De carácter mediterráneo continental, el clima del Prepirineo es más suave y húmedo que el del centro del 
Valle del Ebro. El encinar, coscojar y la sabina albar (Juniperus thurifera) conforman una vegetación potencial intensamente desplazada por los cultivos o degradada hacia matorrales de jara (Cistus ladanifer), retama (Retama sphaerocarpa) o romero (Rosmarinus officinalis).

El Valle Medio del Ebro se caracteriza por su marcada aridez, amplificada por la acción de vientos desecantes, fríos en invierno y frescos en verano. A este ambiente mediterráneo continental muy seco con tendencia a subdesértico, se adapta la sabina albar y el coscojar. En las áreas endorreicas de la cuenca del Ebro en ambientes hidrófilos, la salinidad es determinante para la caracterización de los hábitats y las especies dominantes.

El Sistema Ibérico, montaña mediterránea con fuerte matiz continental, presenta precipitaciones menores que el Pirineo. Aquí se registran las temperaturas más bajas de toda la Península. En cuanto a la vegetación, el quejigo ha desplazado a la encina en un ambiente cultivado o muy degradado hacia matorrales. La sabina albar responde al frío intenso, pero predomina la expansión del pinar de silvestres, rodenos (Pinus pinaster) y laricios (Pinus nigra). La vegetación del somontano del Ibérico se encuentra muy influida por la altitud, que en las zonas elevadas le proporciona una pluviometría mayor. Las temperaturas son ligeramente más bajas y las precipitaciones inferiores a las del somontano pirenaico (Sampietro, 2000). Excepto la cabra doméstica asilvestrada, el resto de ungulados silvestres son especies cinegéticas en Aragón. El territorio se halla acotado para la práctica cinegética en un $91 \%$ de su superficie.

\section{Métodos}

En 2006 llevamos a cabo una encuesta a los Agentes de Protección de la Naturaleza (APN) del Gobierno de Aragón ( $n=242$ encuestas), en la que se consignaron la presencia o ausencia de ungulados silvestres en cuadrículas UTM de 10 x $10 \mathrm{~km}$ en Aragón durante el quinquenio anterior (2001-2005) (Osuna et al., 2006-08). Esta encuesta se repetirá cada cinco años.

Los fareos nocturnos, para establecer la abundancia de ciervos, comenzaron en 1985 en la Reserva de Caza de Montes Universales (RCMU) y a mediados de los 90 se incorporaron los tres grandes núcleos restantes: Reserva de Caza de la Garcipollera (RCG) y alrededores, Montes de Agüero; cotos del Bajo Aragón y la zona adyacente a la RCMU (Figura 1). Los cuatro núcleos monitorizados son objeto de seguimientos mediante muestreos directos nocturnos realizados desde vehículos todo terreno provistos de foco manual, con un mínimo de dos observadores por vehículo, utilizando para el cálculo de densidades el estimador de Kelker (Tellería, 1986) y con una velocidad de 


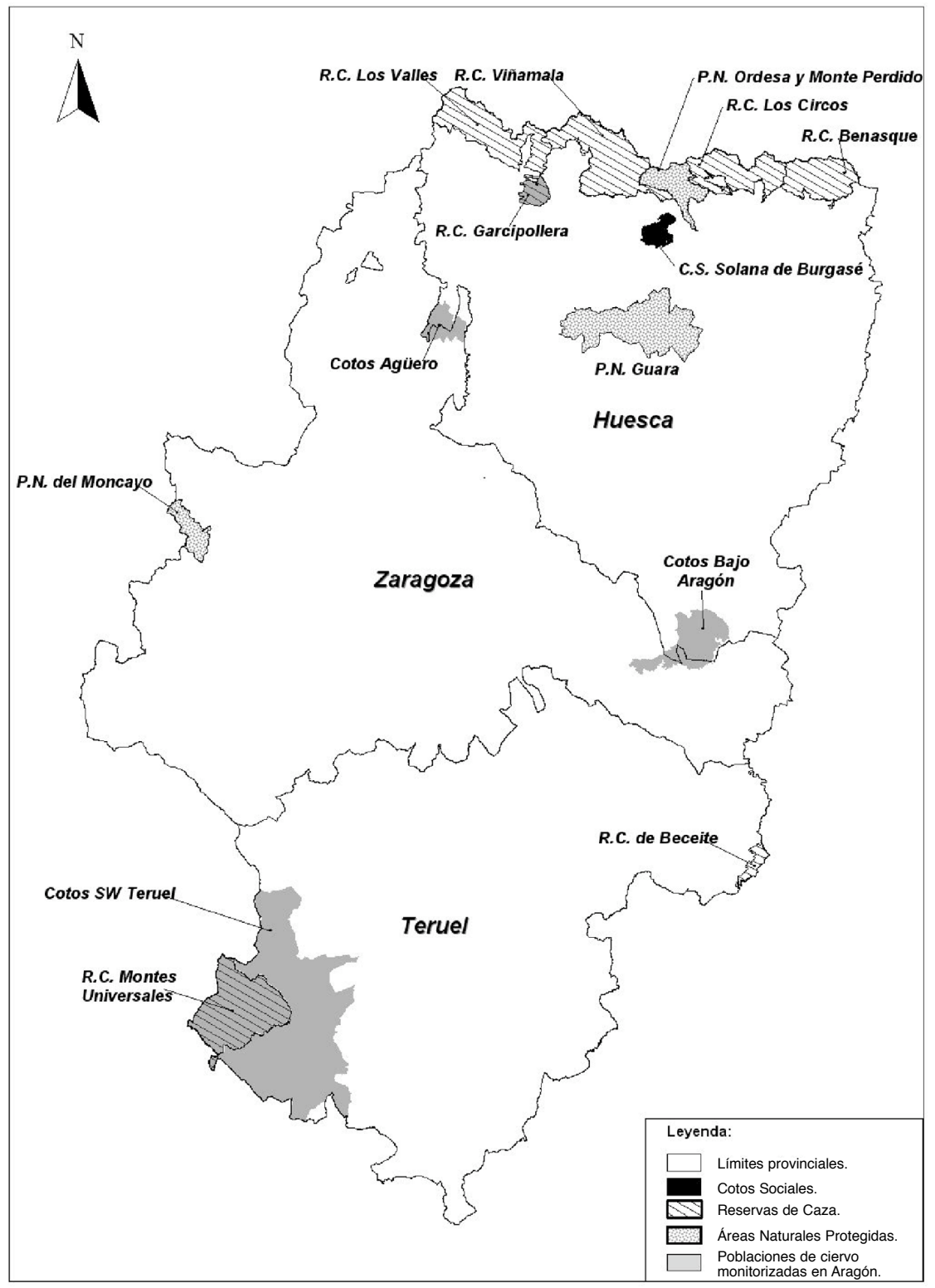

Figura 1. Aragón y las diferentes áreas de seguimiento de poblaciones de ungulados. Corzos y cabras montesas son objeto de seguimiento en toda su área de distribución (ver fig. 2).

Figure 1. Aragon and its different ungulate monitoring areas. Roe deer and Iberian wild goats are monitored all over their distribution area (see fig. 2). 
unos $20 \mathrm{~km} \mathrm{~h}^{-1}$. Cada núcleo se muestreó dos veces en abril y dos en septiembre, época de berrea, por equipos de entre cuatro y doce personas, dependiendo de las dimensiones del territorio.

En la RCMU, RCG y Montes de Agüero se realizan estimas de abundancia a partir de muestreos indirectos basados en el seguimiento de parcelas fijas de acumulación fecal ( $\mathrm{n}=139)$ cada 3-4 meses según zonas (Marco et al., 2005). El método consiste en contar el número de grupos de excrementos que se han acumulado durante un tiempo controlado y en una superficie conocida y constante (que se limpia tras cada revisión), conocida la tasa de defecación de la especie "diana" en el medio concreto que interesa al estudio (Garin, 2000).

El conteo directo en sarrio y cabra montesa se lleva a cabo en toda el área de distribución de las dos especies, a partir de recorridos a pie (mayoritariamente), puntos fijos de observación, recorridos en vehículo y, anecdóticamente, en barca. Los conteos se realizan dos veces al año tras la paridera y en el celo (sarrio); cuatro veces (antes de la paridera, tras ella, antes del celo y en el celo) en la cabra montesa del Parque Natural de los Cañones y Sierra de Guara (PNG) y Zaragoza (Herrero et al., 2004; Herrero et al., 2007; García-Serrano et al., 2010a), dos veces (celo y primavera) en el Alto Matarraña (Teruel) (Marco et al., 2004-2010) y una vez (celo) en el resto de la mitad oriental de la provincia de Teruel (Servicio Provincial de Medio Ambiente de Teruel, 1994-2009). El seguimiento afecta a la totalidad de la población de sarrio; de cabra en Huesca y Zaragoza, y la mayor parte de la población de cabra en Teruel.

Durante la década de los noventa, los estudios sobre el corzo en Aragón estuvieron reducidos a verificar la presencia de la especie en parajes cada vez más numerosos. A partir de 2002 se inició un seguimiento de la abundancia de la especie en toda la comunidad autónoma, abarcando 8 grandes unidades territoriales de entre 150.000 y $450.000 \mathrm{ha}$, a partir de transectos de recuento de grupos fecales, para realizar los muestreos a razón de una o dos unidades/año, según sus dimensiones. Como índice de abundancia se ha calculado la densidad de grupos fecales, estimada a partir de transectos de anchura de banda variable (Acevedo et al., 2010). Estos datos se han transformado en densidad de corzo a partir de una tasa de defecación de 20 grupos fecales día ${ }^{-1}$ (Mayle et al., 1999) y un periodo de persistencia medio de 135 días (Escudero et al., 2007) y dentro del rango de entre 116 y 164 días estimado en Grecia por Tsaparis et al., (2009). Hasta 2009 se han establecido un total de 332 puntos de muestreo con dos transectos de $700 \mathrm{~m}$ cada uno, estando previsto llegar casi a los 500 puntos cuando se completen las ocho unidades definidas para toda la comunidad autónoma. En los espacios donde existen parcelas fijas de conteo de excrementos orientadas a ciervo, se obtienen resultados de corzo que enriquecen la precisión de las estimas de abundancia de esta especie a nivel más local. 
El método de las batidas cinegéticas de jabalí consiste en calcular la superficie batida durante su caza así como el número de jabalíes y otros animales que son vistos durante la misma (Herrero, 2003). Ha sido utilizada en las Reservas de Caza del Pirineo aragonés (Herrero et al., 2008) y en el Parque Natural del Moncayo (PNM) (Giménez-Anaya et al., 2009).

El muestro de distancias o distance sampling, está basado en el cálculo de la distancia a la que es avistado un ejemplar con respecto al observador, en puntos o en transectos de muestreo (Buckland et al., 2001). Esta segunda aproximación se ha llevado a cabo para el sarrio durante la estima poblacional de bucardo Capra p. pyrenaica (García-González et al., 1992) en el Parque Nacional de Ordesa y Monte Perdido, así como en el mismo macizo, como parte del seguimiento anual del sarrio desde 1998 y en el corzo en las Reservas de Caza pirenaicas desde 2002 y en el PNM desde 2009. La cabra doméstica asilvestrada también ha sido estimada a partir del muestreo de distancias en el PNG en 1995, a partir de transectos (Gortázar et al., 1997) y puntos fijos de observación (Herrero et al., 2009a) y lo será de esta forma quinquenalmente.

Las capturas cinegéticas (1999-2009, 11 años) se calculan a partir del número de ejemplares que los titulares de los terrenos cinegéticos (sociedades de cazadores, ayuntamientos, propietarios privados o administración autonómica) declaran haber capturado durante la temporada cinegética. Generalmente en estas declaraciones no se incluyen las capturas obtenidas fuera de temporada en controles excepcionales por daños agrícolas u otras causas. Todos los ungulados cinegéticos menos el jabalí y el gamo son objeto de caza a partir de cupos determinados por la Administración para cada territorio cinegético. Cada ejemplar cazado es precintado a excepción, nuevamente, del jabalí.

\section{Resultados}

El jabalí ocupa la totalidad del territorio aragonés (Figura 2), con densidades no muy dispares (Tabla 1) y sus capturas se han triplicado, siendo la especie con mayor distribución y capturas (83\% en 2009) (Tabla 5).

El ciervo, ocupa el 30,1\% del territorio (Figura 2) y sus capturas se han triplicado también (Tabla 5) (Marco et al., 2010). Las mayores poblaciones se encuentran en la RCMU y sus cotos colindantes (Cotos SW prov. Teruel), seguidas de los Cotos del Bajo Aragón, la RCG y Agüero, en orden decreciente. La mayor densidad aparece en la RCG. Considerando los cuatro núcleos existentes y la titularidad de los terrenos, los que son gestionados por la Administración autonómica presentan mayores densidades (Tabla 2). Los cupos de capturas han podido reducir el tamaño poblacional en la Reserva de Caza de La Garcipollera, tras una intensa presión cinegética en un espacio de 

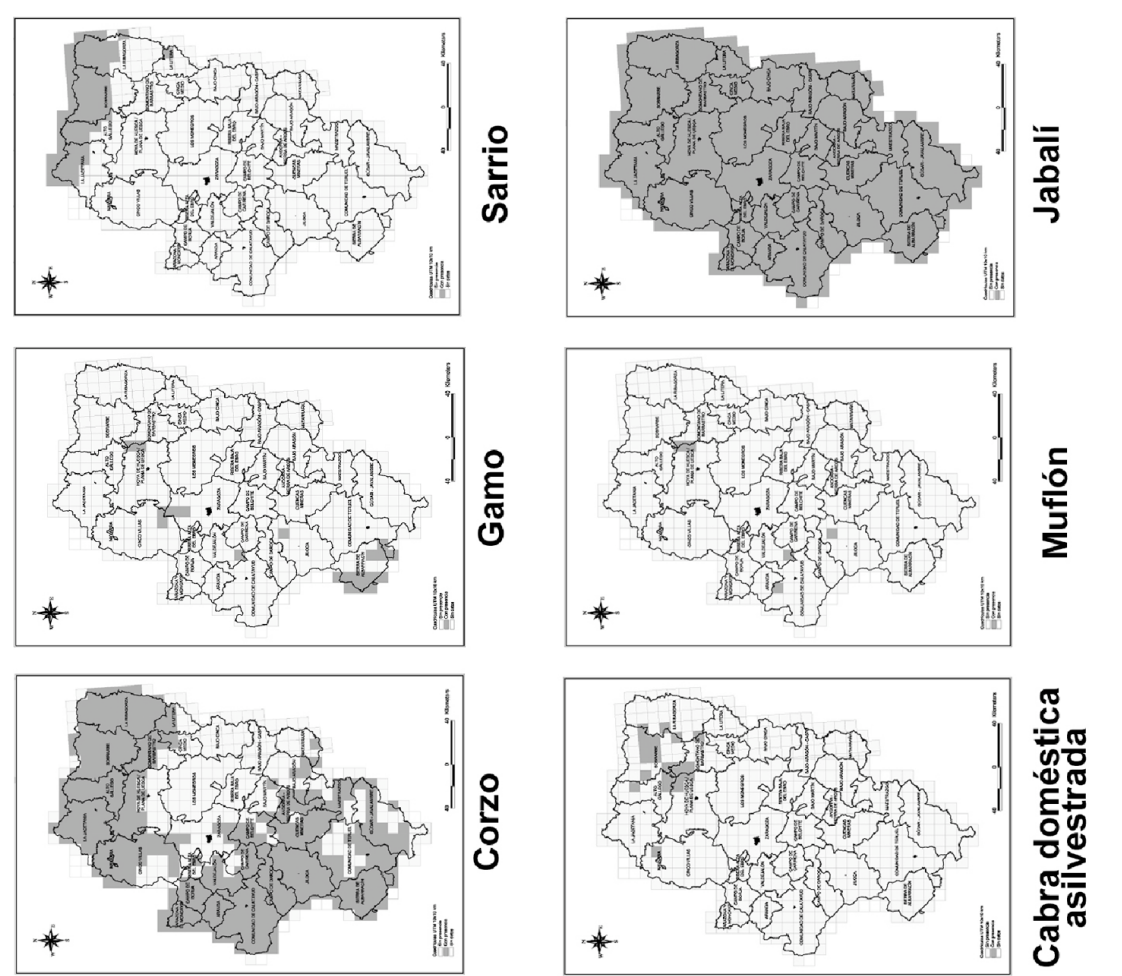

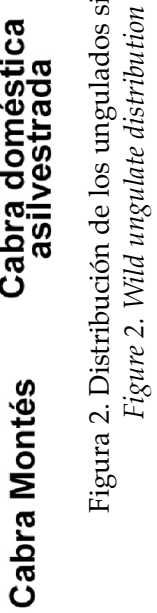
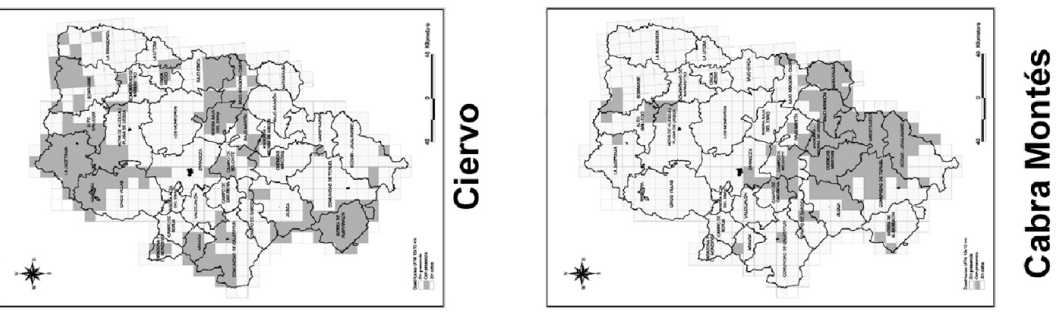
VEINTE AÑOS DE SEGUIMIENTO POBLACIONAL DE UNGULADOS SILVESTRES DE ARAGÓN
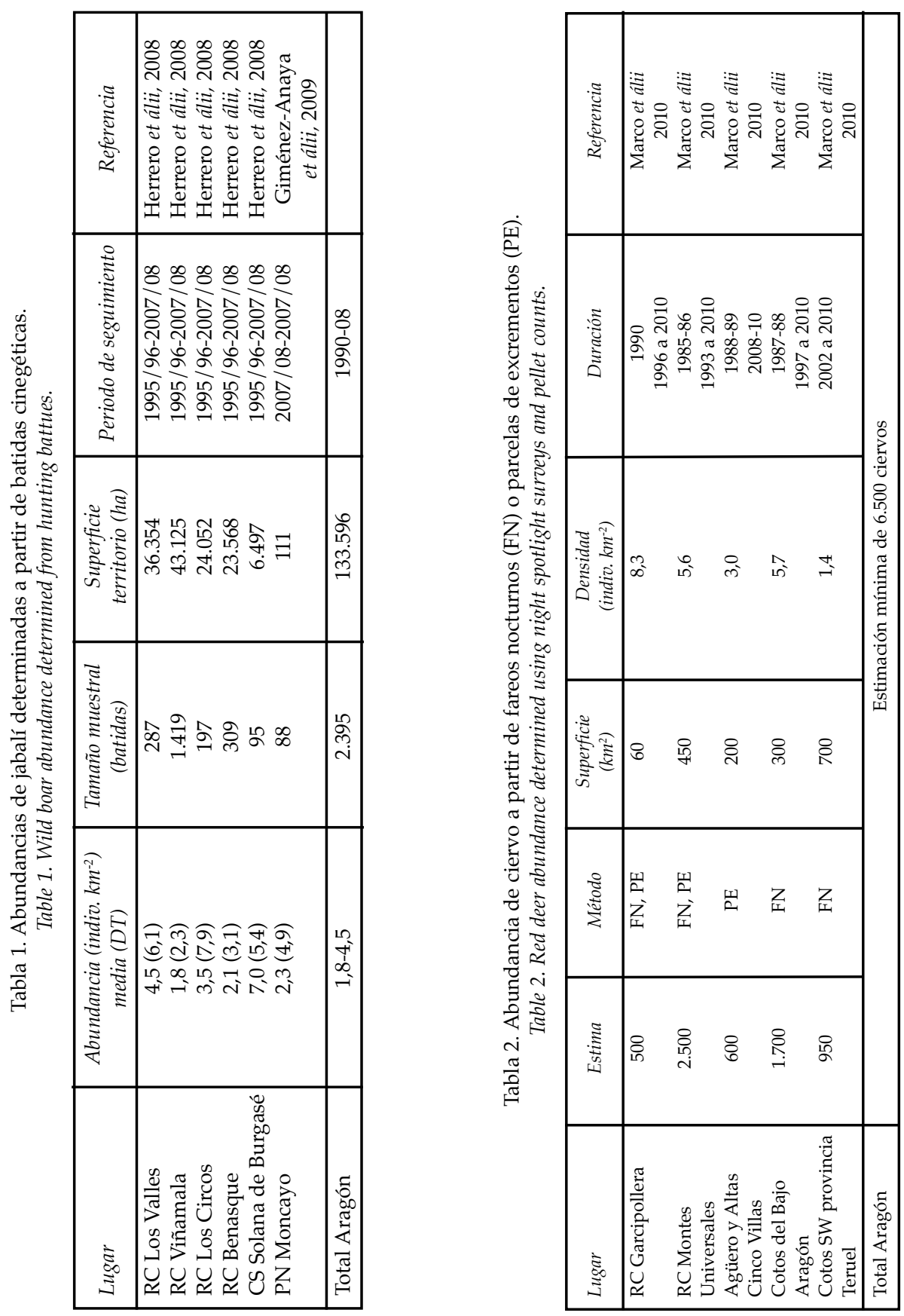

(Pirineos, 2011, Vol. 166, 135-153, ISSN 0373-2568, eISSN: 1988-4281, doi: 10.3989/Pirineos.2011.166007) 
J. MARCO et al.
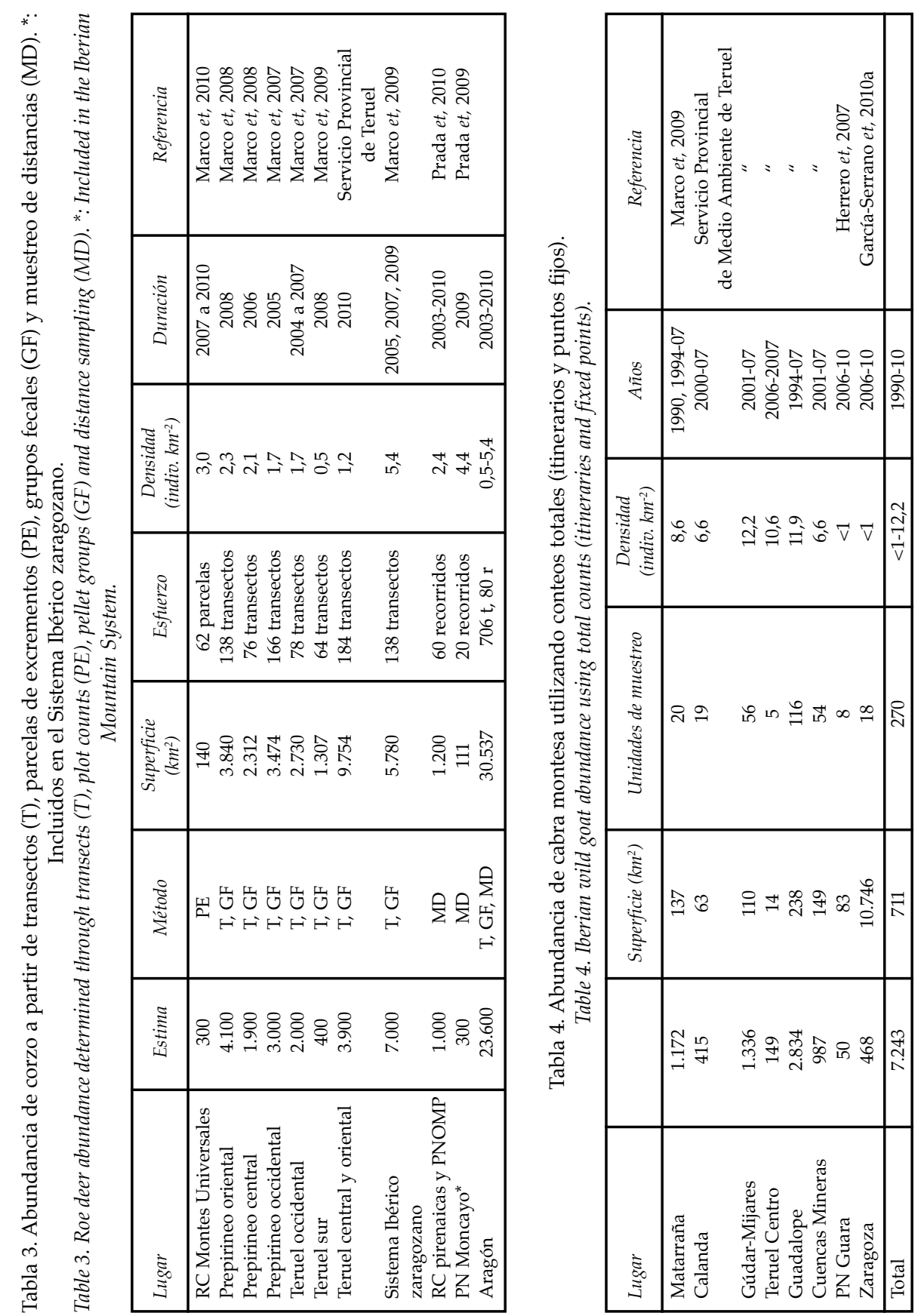

144 (Pirineos, 2011, Vol. 166, 135-153, ISSN 0373-2568, eISSN: 1988-4281, doi: 10.3989/Pirineos.2011.166007) 
VEINTE AÑOS DE SEGUIMIENTO POBLACIONAL DE UNGULADOS SILVESTRES DE ARAGÓN

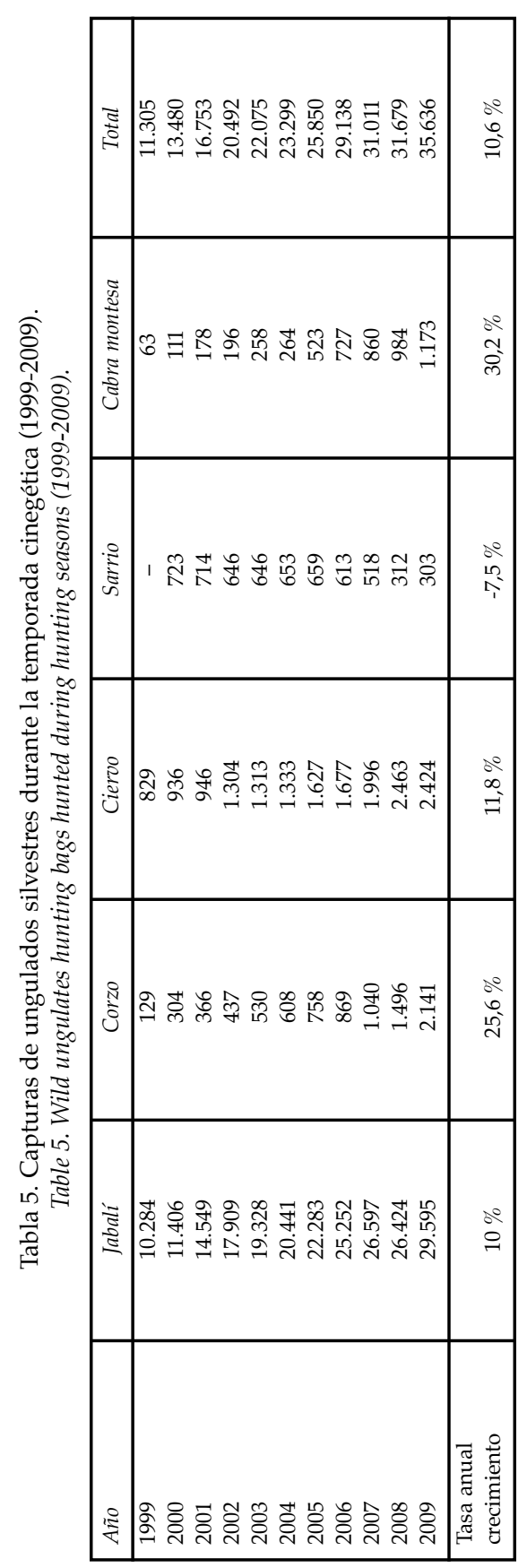


discreta extensión (5.580 ha). La expansión territorial y demográfica parece asimismo contenida en las poblaciones prepirenaicas occidentales.

El corzo está presente en el 59,3\% del territorio (Figura 2) y presenta abundancias dispares (Tabla 3). Sus capturas se han multiplicado por 16,6 (19992009), si bien se parte de unos cupos asignados con poca información en 1999 (129 ejemplares). A partir de 2007, la información disponible es más precisa y afecta a gran parte de la comunidad autónoma, pasando los cupos de 1.040 a 2.141 ejemplares en 2009, esto es duplicando el cupo (Tabla 3) (Ferreres et al., 2010).

La cabra montesa ocupa el 23,2\% del territorio (Figura 2). En Huesca la subespecie autóctona Capra p. pyrenaica se extinguió en 2000 (García-González $\&$ Herrero, 2000). Desde mediados de los 90 un escape de cabras pertenecientes a la subespecie C. p. hispanica procedentes de Cazorla, comenzaron a repoblar el PNG. En 2006 comenzó su seguimiento y en 2010 su población se estima en no menos de 50 ejemplares en pleno proceso de expansión (Herrero et $a l ., 2007)$. En Zaragoza la presencia de la cabra montés comienza hacia principios de los 90 como resultado de la expansión natural de la especie desde Teruel. En 2006 comienza su seguimiento y en 2010 se estiman un mínimo de 300 cabras para toda la provincia, también en pleno proceso de expansión (García-Serrano et al.,, 2010a). En Teruel y a partir de 1994, se inicia una rutina de estimas poblacionales, en años alternos, en época de celo, por medio de recorridos y puntos de observación, en unidades previamente diseñadas e identificadas cartográficamente (Informes inéditos, Gobierno de Aragón). A medida que la especie ha colonizado nuevos territorios se ha ido ampliando la superficie de estudio hasta alcanzar las casi cien mil ha actuales. Se estimaron unas 7.000 cabras en 2007, aunque no todas las subpoblaciones son objeto de seguimiento. A partir de 2004 se estandarizan y generalizan los seguimientos en gran parte de Teruel, de forma que se llega a proponer una extracción de 1.173 ejemplares en 2009; esto es, en cinco años el cupo se ha multiplicado por 4,5 (Tabla 4).

El sarrio ocupa la práctica totalidad de su área potencial (Figura 2). Su población se ha estimado a partir de conteos totales de forma puntual desde 1983. Desde entonces diversos trabajos han ido aproximándose al tamaño de sus poblaciones. En 1995-96 comienza un seguimiento anual propiamente dicho de forma coordinada entre Aragón y Navarra (Herrero et al., 2010) y en 2000 todo el conjunto de subpoblaciones están integradas en él. Desde entonces pueden distinguirse tres periodos en cuanto a su tendencia: incremento moderado (2000-2007); decremento por una epidemia de queratoconjuntivitis infecciosa (2007-2009) y comienzo de la recuperación (2010) (Figura 3). Esto ha llevado parejo la adecuación del cupo, que en ausencia de brotes epidémi- 
cos con mortalidad relevante rondan el 5\%. (Tabla 5). En 2010 el número mínimo de ejemplares se estima en unos 13.000 (García-Serrano et al., 2010b).

La cabra doméstica asilvestrada ocupa tan solo el 3,6\% del territorio aragonés (Figura 2) y está presente en el Pirineo y Prepirineo. El conjunto de su población no ha sido estimado, aunque en el PNG su número asciende a 941 animales y su densidad a 2,1 cabras $\mathrm{km}^{-2}$ (Herrero et al., 2009), lo que coincide con lo estimado en 1995 por Gortázar et al., (1997).

La presencia de arruí se circunscribe en Aragón al coto cercado de Bastarás, en el PNG (Figura 2), en el que en 2009 había alrededor de unos 30 ejemplares (Herrero et al., 2009b). El muflón está también circunscrito a este cercado y algún otro, no manteniendo poblaciones viables fuera de los mismos. El gamo también tiene una presencia marginal, circunscrita a la RCMU $\mathrm{y}$ alrededores y procedente de algunos escapes (Figura 2).

\section{Discusión}

Todos los ungulados silvestres presentes en Aragón tienen una distribución conocida y los de abundancia relevante son objeto de seguimiento demográfico en la práctica totalidad de su área de distribución. La extraordinaria recuperación de la comunidad de ungulados aragonesa desde mediados del siglo XIX hasta finales del XX (Madoz, 1848; Gortázar et al., 2000) probablemente continuará en el futuro, lo cual plantea un escenario muy dinámico en el que la monitorización de las poblaciones es crucial para su gestión. El papel esencial lo juegan afortunadamente las especies autóctonas, siendo las especies y poblaciones introducidas bastante marginales, aunque el papel de la cabra doméstica asilvestrada posee cierta importancia (Aldezabal \& Garin, 2000). Salvo en el caso del sarrio, con poblaciones bastante estabilizadas o fluctuantes y un área potencial ya ocupada, el incremento en las capturas de las cuatro especies principales (jabalí, ciervo, corzo, cabra montesa) muestra (Figura 3), por un lado, su recuperación, y por otro, la necesidad de controlar su abundancia y expansión, en plena fase inicial de incremento; algo evidentemente difícil y que requiere un notable esfuerzo.

Esta expansión, unida a los cambios de cultivo de secano a regadío (cereales y frutales) en amplias zonas del territorio, está planteando un nivel de incompatibilidad entre la agricultura y la recuperación de los ungulados silvestres que muestra un nuevo escenario: la necesidad de zonificar el territorio en función de la capacidad de carga social de estas especies, influida de forma determinante por la relevancia de los daños agrícolas y los atropellos de vehículos. En pocos años, el modelo ha pasado del fomento de la conser- 
vación, a la promoción del control de muchas especies, tal y como ocurre en el resto de Europa (Apollonio et ál., 2010; Ferreres et ál., este mismo número).

Las poblaciones de ungulados silvestres han crecido y colonizado nuevos territorios, no sólo por el seguimiento de criterios de extracción conservadores y orientados a la promoción poblacional, sino por la creciente disponibilidad de superficies con buenas condiciones de refugio y tranquilidad, consecuencia del abandono de usos tradicionales y de la presencia humana en zonas de montaña y terrenos con rendimientos marginales; lugares en los que la caza mayor se ha convertido en el aprovechamiento rústico mejor pagado, muy por encima del pastoril o del forestal. Sin embargo, en las zonas en las que se ha modificado recientemente la vocación de algunos cultivos, gracias a la creación de nuevos regadíos, las especies de caza mayor son rechazadas por el agricultor, el cual no asume la preexistencia de éstas y los condicionantes que conlleva su presencia en lo que concierne a medidas de protección de cultivos, pretendiendo que sea el sector cinegético o la Administración quienes satisfagan sus pérdidas o costeen dichas medidas de protección, incluyendo la eliminación de la especie problema.

Los cupos de caza han sido claramente conservadores con carácter general en toda la Comunidad Autónoma, si bien, debido a la conflictividad con los productores de cultivos leñosos (principalmente cerezos y viñas) en el Sistema Ibérico zaragozano, desde 2008 se vienen aplicando tasas de extracción mucho más severas en estas zonas.

El periodo de caza del jabalí no ha hecho más que ampliarse y no existen apenas condicionantes para autorizar batidas y esperas por daños fuera de temporada. Podría afirmarse, con independencia de las variaciones locales de la abundancia, que el número de jabalíes cazados depende de la capacidad y dedicación de los cazadores ya que éstos no tienen limitadas las capturas, pese a lo cual éstas no han hecho más que crecer en los últimos once años (Figura 3).

La población de ciervo del Bajo Aragón ha visto duplicados sus efectivos en diez años pese a la presión cinegética ejercida, lo cual, junto a la creciente implantación de nuevos regadíos en la zona, ha hecho necesario casi duplicar la tasa de extracción en las dos últimas temporadas de caza, en pos de una drástica reducción del tamaño poblacional a corto plazo. En la Reserva de Caza de Montes Universales la importante presión de caza que se mantiene desde hace cinco años, tan sólo consigue contener la explosión demográfica de la especie, sin evitar un discreto pero constante crecimiento, habida cuenta del importante flujo de animales que se produce con las vecinas Cuenca y Guadalajara, en las que los criterios de gestión poblacional no tienen por qué coincidir con los seguidos en dicha Reserva. 


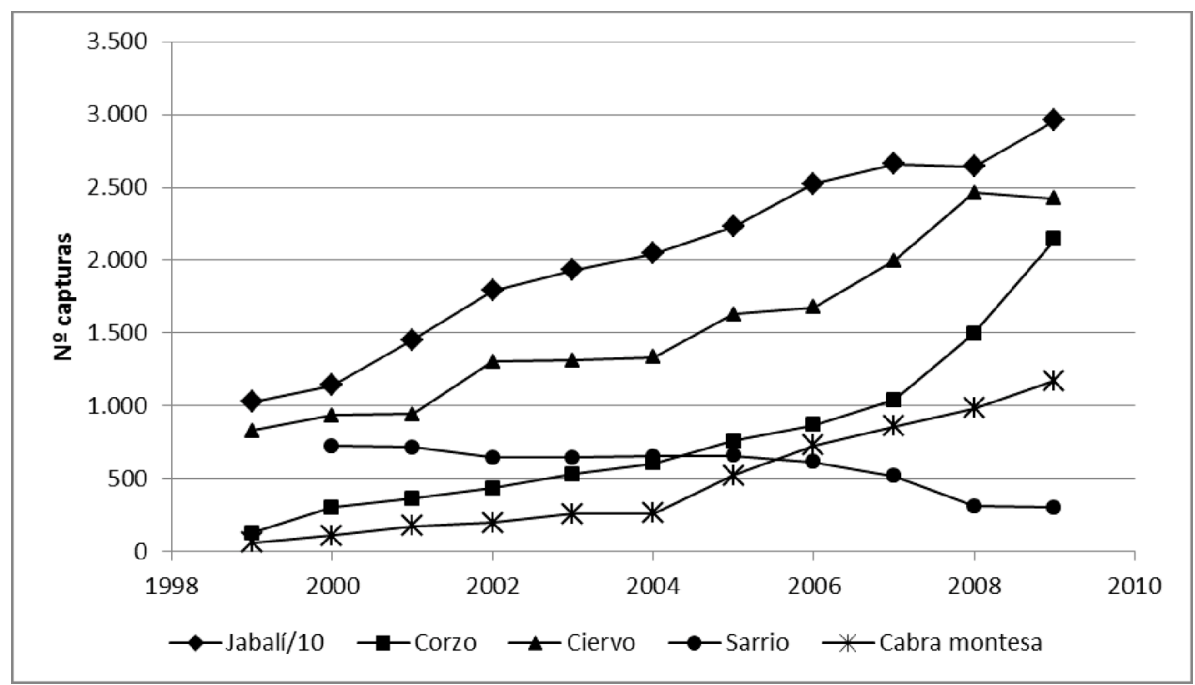

Figura 3. Evolución del número de capturas de ungulados silvestres en Aragón. Figure 3. Evolution of the number of hunted wild ungulates in Aragon.

Los bajos cupos de corzo han permitido un evidente crecimiento de la abundancia y expansión territorial de la especie, sobre todo en las zonas de reciente ocupación en las que no existía tradición para su caza. Por el contrario, en las zonas donde las cabras monteses son objeto de caza desde hace décadas (Matarraña y Maestrazgo), los titulares de cotos valoran la importancia económica de este recurso y la necesidad de llevar una rigurosa gestión cinegética, hallándose más limitado el crecimiento poblacional.

De forma creciente, se están produciendo sentencias judiciales que obligan a los cotos de caza a fuertes desembolsos en concepto de indemnizaciones por daños agrícolas y colisiones con vehículos provocados por ungulados cinegéticos. Ello puede animar a los titulares de cotos, de hecho ya existen precedentes, a solicitar la anulación de los mismos por no poder afrontar estos pagos. No hay que ignorar que el cazador está dispuesto a pagar una cuota, a veces importante, por satisfacer una afición en su tiempo de ocio; en modo alguno le compensará triplicar dicha cuota para afrontar el coste de la alimentación de los ciervos (que a lo mejor nunca tiene suerte de cazar) o de las reparaciones de vehículos que colisionan con jabalíes. Un efecto contagio entre titulares de cotos para renunciar a éstos y dejar el territorio sin cazar, puede conllevar a medio plazo la explosión demográfica de algunas poblaciones de ungulados silvestres, salvo que la Administración introdujera con- 
troles con medios propios o externos, con el coste que ello podría suponer. Cierto es que llegados a situaciones de sobreabundancia, las poblaciones podrían sufrir la acción de mecanismos de regulación dependientes de la densidad, pero mientras eso ocurre ¿Soportarían los particulares o el erario público un crecimiento geométrico de los daños?

Sea como fuere, parece evidente que el ejercicio de la caza mayor, sin perder su componente de actividad lúdica, con cierta repercusión económica para los propietarios de terrenos cinegéticos y otros sectores, se ha convertido en una herramienta de control de ciertas poblaciones de ungulados silvestres (al uso de lo que ocurre con el conejo de monte allí donde es muy abundante). La adecuada utilización de esta herramienta, va a ser de gran utilidad para mantener dichas poblaciones en niveles compatibles con su propia conservación, manteniendo unas afecciones sobre intereses antrópicos que resulten soportables o asumibles, por quienes las padecen y por quienes deban satisfacer su coste.

\section{Agradecimiento}

Los trabajos que han servido de base a este artículo son mayoritariamente resultado de asistencias técnicas financiadas por el Gobierno de Aragón. Agradecemos el esfuerzo llevado a cabo por los Agentes de Protección de la Naturaleza, vigilantes contratados y técnicos del Gobierno de Aragón. Los seguimientos poblacionales de ungulados comenzaron a mediados de los años ochenta en Aragón, gracias al empeño de personas como Julio Guiral y Matilde Cabrera, técnicos ambos del Gobierno de Aragón. Posteriormente otros responsables administrativos en materia cinegética desde las reservas de caza, unidades de caza y pesca de las tres provincias y sucesivas direcciones generales competentes en la materia, han continuado con la labor de promover y coordinar estos seguimientos, por encima de no pocas adversidades presupuestarias y de la frecuente incomprensión de propios y extraños ante la utilidad de tanto esfuerzo. Vaya para todos ellos nuestro más sincero reconocimiento.

\section{Referencias}

Acevedo, P., Ferreres, J., Jaroso, R., Durán, M., Escudero, M.A., Marco, J. \& Gortázar, C., 2010. Estimating roe deer abundance for pellet groups counts in Spain: an assessment of methods suitable for Mediterranean woodlands. Ecological indicators, 10: 1226-1230. 
VEINTE AÑOS DE SEGUIMIENTO POBLACIONAL DE UNGULADOS SILVESTRES DE ARAGÓN

Aldezabal, A. \& Garin, I., 2000. Browsing preferences of feral goats (Capra hircus L.) in a Mediterranean mountain scrubland. Journal of Arid Environments, 44 (1): 133-142.

Apollonio, M., Anderson, R. \& Putman, R., 2010. European Ungulates and their Management in the $21^{\text {st }}$ Century. Cambridge University Press, Cambridge (Reino Unido).

Buckland S.T., Anderson, D.R., Burnham, K.P., Laake, J.L., Borchers, D.L. \& Thomas, L., 2001. Introduction to Distance Sampling. Oxford University Press, Oxford, Reino Unido.

De la Riva, J., 1997. Atlas Geográfico de Aragón en Internet. Disponible en http: / / 155.210.60.15/geoatlas / atlas.htm (consulta: julio de 2006).

Escudero, M.A., Ferreres, J. \& Marco, J., 2007. Situación de la población de corzo en el Parque Natural del Moncayo. Informe interno del Gobierno de Aragón. Zaragoza.

Ferreres, J., Escudero, M.A., Marco, J. \& Escudero, E., 2011. Gestión del corzo en Zaragoza: de conservación a control poblacional. Pirineos, 166: 69-86.

García-González, R. \& Herrero, J., 1999. El bucardo de los Pirineos: historia de una extinción. Galemys, 11: 17 -26.

García-González, R., Hidalgo, R., Ameztoy, J.M. \& Herrero, J., 1992. Census, population structure and habitat use of a chamois population in Ordesa N.P. living in sympatry with Pyrenean wild goat. Proceedings of the Symposium Ongulés/Ungulates 91. F. Spitz, G. Janeau, G. Gonzalez, S. Aulagnier, Eds.: 321-25.

García-Serrano A., Prada, C., Fernández-Arberas, O. \& Herrero, J., 2010. Seguimiento de la cabra montesa en Zaragoza durante 2010. Informe interno del Gobierno de Aragón. Zaragoza.

García-Serrano, A., Prada C., Fernández-Arberas, O. \& Herrero, J., 2010b. Seguimiento de los ungulados en el Pirineo aragonés. Informe interno del Gobierno de Aragón. Zaragoza.

Garín, I., 2000. El ciervo de la Reserva de Caza de la Garcipollera. Publicaciones del Consejo de Protección de la Naturaleza de Aragón, serie Investigación $n^{\circ} 22$.

Giménez-Anaya, A., Revilla, M., Prada, C., Arnal, M.C., García-Serrano, A., Martínez, D., Fernández de Luco, D. \& Herrero, J., 2009. El jabalí Sus scro$f a$ y su caza en el Parque Natural del Moncayo. Un ejemplo de seguimiento poblacional en un espacio natural protegido. Naturaleza Aragonesa, 22: 20-25.

Gortázar, C., Ferreres, J., Marco, J., Fernández de Luco, D., Garin, I. \& Aldezabal, A., 1997. The feral goat in Guara (Aragón, Spain): conservation or erradication. BIPAS, 17: 73-79. 
Gortázar, C., Herrero, J., Villafuerte, R. \& Marco, J., 2000. Historical examination of the status of large mammals in Aragon, Spain. Mammalia, 64: 411422.

Herrero, J., 2003. Adaptación funcional del jabali a un ecosistema forestal y a un sistema agrario intensivo en Aragón. Publicaciones del Consejo de Protección de la Naturaleza de Aragón. Serie Investigación, número 41. 159 páginas.

Herrero, J., Escudero, E., García, J.M., García-Serrano, A., Prada, C. \& Couto, S., 2004. Gestión y seguimiento demográfico del sarrio en el Pirineo aragonés. En: J. Herrero, E. Escudero, D. Fernández de Luco, R. GarcíaGonzález (Eds.). El sarrio pirenaico Rupicapra p. pyrenaica: biología, patología y gestión. Actas de las Primeras Jornadas sobre el Sarrio Pirenaico. Publicaciones del Consejo de Protección de la Naturaleza de Aragón. Serie Investigación, número 46, 263 páginas. Páginas: 69-82.

Herrero, J., Garin, I., Prada, C. \& García-Serrano, A., 2010. Interagency coordination fosters the recovery of the Pyrenean chamois Rupicapra p. pyrenaica at its western limit. Oryx, 44 (4): 529-532.

Herrero, J., Giménez-Anaya, A., García-Serrano, A., Prada, C. \& FernándezArberas, O., 2008. Seguimiento del jabalí Sus scrofa L. en las Reservas de Caza de Los Valles, Viñamala, Los Circos y Benasque y el Coto Social de la Solana de Burgasé. Informe interno del Gobierno de Aragón. Zaragoza.

Herrero, J., Prada, C., Fernández-Arberas, O. \& García-Serrano, A., 2009a. Estimación poblacional de la cabra doméstica asilvestrada Capra hircus en el Parque Natural de la Sierra y Cañones de Guara durante 2009. Informe interno del Gobierno de Aragón. Zaragoza.

Herrero J., Prada, C., Fernández-Arberas, O. \& García-Serrano, A., 2009b. El arrui Ammotragus laervia en el Parque Natural de la Sierra y Cañones de Guara. Informe interno del Gobierno de Aragón. Zaragoza.

Herrero, J., Prada, C., Fernández-Arberas, O. \& García-Serrano, A., 2007. The beginning of the recovery of the Iberian wild goat Capra pyrenaica in the Pyrenees. Newsletter of the IUCN/SSC Caprinae Specialist Group, Marzo 2007: 6-7.

Madoz, P., 1845-1850. Diccionario geográfico-estadístico-histórico de España y sus posesiones de ultramar. La Ilustración, Madrid. [Edición facsímil a cargo de Sánchez Zurro, D. (ed.). Diccionario geográfico-estadístico-histórico: Aragón. 3 volúmenes. Ámbito Ediciones S. A., Valladolid. 307pp. Año 1985.

Marco, J., Escudero, M.A., Ferreres, J., Labarta, J.L. \& Monrabal, L., 1996-2010. Censo de las poblaciones de ciervo en Aragón: Propuesta de Plan de Caza para las temporadas de caza 1996-2010. Informes internos del Gobierno de Aragón. Zaragoza.

Marco, J., Escudero, M.A., Labarta, J.L. \& Monrabal, L., 2005. Determinación del tamaño de la población de ciervo (Cervus elaphus l.) en la Reserva de Caza de la 
Garcipollera (Huesca) mediante métodos de censo indirectos. Informe interno del Gobierno de Aragón. Zaragoza.

Marco, J. \& Jordán, G., 1990. Estudio de la población de corzo en el Macizo del Moncayo y área de influencia. Informe interno del Gobierno de Aragón.

MArco, J., Ferreres, J., Escudero, M.A., Labarta, J.L. \& Monrabal, L., 20042010. Estimación de abundancias de corzo en Aragón según sectores entre 2004 y 2010. Informes internos del Gobierno de Aragón. Zaragoza.

Marco, J., Escudero, M.A., Ferreres, F., Labarta, J.L. \& Monrabal, L., 2009-2010. Seguimiento de la población de cabra montés en el núcleo poblacional del Alto Matarraña: Propuesta de capturas temporada 2009-2010. Informe interno del Gobierno de Aragón. Zaragoza.

Mayle, B.A., Peace, J.A. \& Gill, R.M., 1999. How many deer? A field guide to estimating deer population size. UK: Forestry Commision, Edingburgh. Field Book 18.

Osuna, D., Prada, C., Herrero, J. \& Marco, J., 2006-2008. Distribución de los ungulados silvestres en Aragón (2001-2005) determinada a partir de encuestas. Lucas Mallada, 13: 191-212.

Prada, C., García-Serrano, A., Fernández-Arberas, O. \& Herrero, J., 2010. Seguimiento del corzo Capreolus capreolus en el Pirineo aragonés. Informe interno del Gobierno de Aragón. Zaragoza.

Prada, C., Herrero, J., Fernández-Arberas, O. \& García-Serrano, A., 2009. Protocolos para el Seguimiento ecológico del Parque Natural del Moncayo. Informe interno del Gobierno de Aragón. Zaragoza.

Sampietro Latorre, F.J., Pelayo Zueco, E., Hernández Fernández, F., Cabrera Millet, M. \& Guiral Pelegrín, J., 2000. Descripción fisiográfica de la Comunidad Autónoma de Aragón. En: Aves de Aragón. Atlas de especies nidificantes. Diputación General de Aragón, segunda edición. 566 páginas.

Tellería, J.L., 1986. Manual para el censo de vertebrados terrestres. Ed. Raíces. Madrid

Tsaparis, D., Katsanevakis, S., Ntolka, E. \& Legakis, A., 2009. Estimating dung decay rates of roe deer (Capreolus capreolus) in different habitat types of a Mediterranean ecosystem: an information theory aproach. Eur. J. Wildlife Res., 55, 167-172. 